\title{
Research on A Kinematic Performance by Interpolation Algorithm of Zadoff-Chu Sequence \\ Jian $\mathrm{CaO}^{\mathrm{a}}$, Gang $\mathrm{Li}^{\mathrm{b}}$, Cong $\mathrm{Yan}^{\mathrm{c}}$
}

\author{
Air Force Engineering University, Xi'an, Shaanxi Province 710051, P.R. China \\ accao_jian1972@163.com, ${ }^{\mathrm{b}} \mathrm{LiGang} @ 163 . c o m,{ }^{c}$ yancong192@qq.com
}

Keywords: design; kinematic performance; CC2430; VCR

\begin{abstract}
There are many factors that can affect the engine network performance, such as delay, packet loss and capacity utilization. Application examples show that, this system can significantly improve the efficiency of aerodynamic design. Compare the kinematics function with the new design with the prototype 250 engine and the traditional multi-link and variable compression ratio mechanism by using the virtual simulation software MSC-Adams. The contrast result suggested that the new design had higher combustion efficiency and charging efficiency than the prototype engine.
\end{abstract}

\section{Introduction}

Currently, with the growing demand for development of space resources and increasingly fierce competition of commercial satellite launch market, the next generation launcher with high-performance and low-cost is becoming research focus in many countries. Modular design is considered to be the future direction of launch vehicle ${ }^{[1]}$. It decomposes the launcher into several standard modules. By combing different modules, various capabilities launch vehicle would be assembled to meet different payload requirements. Therefore, the development cycle will be significantly shortened, the costs will be lower, and reliability will be enhanced. Aerodynamic performance of launch vehicle mainly depends on their shape exposed to the air. It also affects other characteristics of launch vehicle, such as trajectory, flight stability, mechanical properties, etc. R\&D of Launch vehicle is facing combinatorial explosion problem caused by modular design. That is to say, the possible number of launch vehicle is much more than the number of modules ${ }^{[2][3]}$.

At present, mankind is facing with serious problem of energy consumption and environmental pollution. As the main carrier of energy consumption and the main factors of city environmental pollution, realizing its energy saving will become increasingly important. Now a lot of technology for achieving low power consumption and low emissions had been developed. Such as variable valve timing (VVT), EGR technology, direct injection lean burn technology, HCCI technology, and variable compression ratio technology (VCR). The concept of VCR has been earlier proposed in the last century, mainly by changing the position of connecting rod up and down, changing the position of the crankshaft, redesigning cylinder head and the cylinder or changing the combustion chamber volume to get continuously variable compression ratio. The main characteristics of this technology is that it can improve fuel economy ${ }^{[4]}$. For gasoline, the using of supercharger and VCR can increase engine efficiency and enhance engine power and improve the emissions. But until now, because of cost, processing, installation and other reasons that this technology is still not used for mass production car. But in the future, this technology will become the main direction of automotive engine development ${ }^{[5]}$.

This paper studies a new design single-cylinder VCR engine with multi link. We use multi-body dynamics software MSC-Adams to create a virtual prototype. And compare its kinematics performance with that of the original machine to illustrate the performance of this new engine, finally we can use the results to guide the bench test of the final prototype.

\section{Hardware Circuit Design}

The Central Processing Unit (CPU) applies the industrial chips of MC9S08 AC16, which are 
industrial eight-digit single-chip microprocessors with ultra-low power consumption. This type of chips internally integrate a 16K-byte FLASH and a1k-byte RAM memory with SPI interface, A/D conversion circuit. System framework based on multi-layer network architecture is shown in Fig.1.

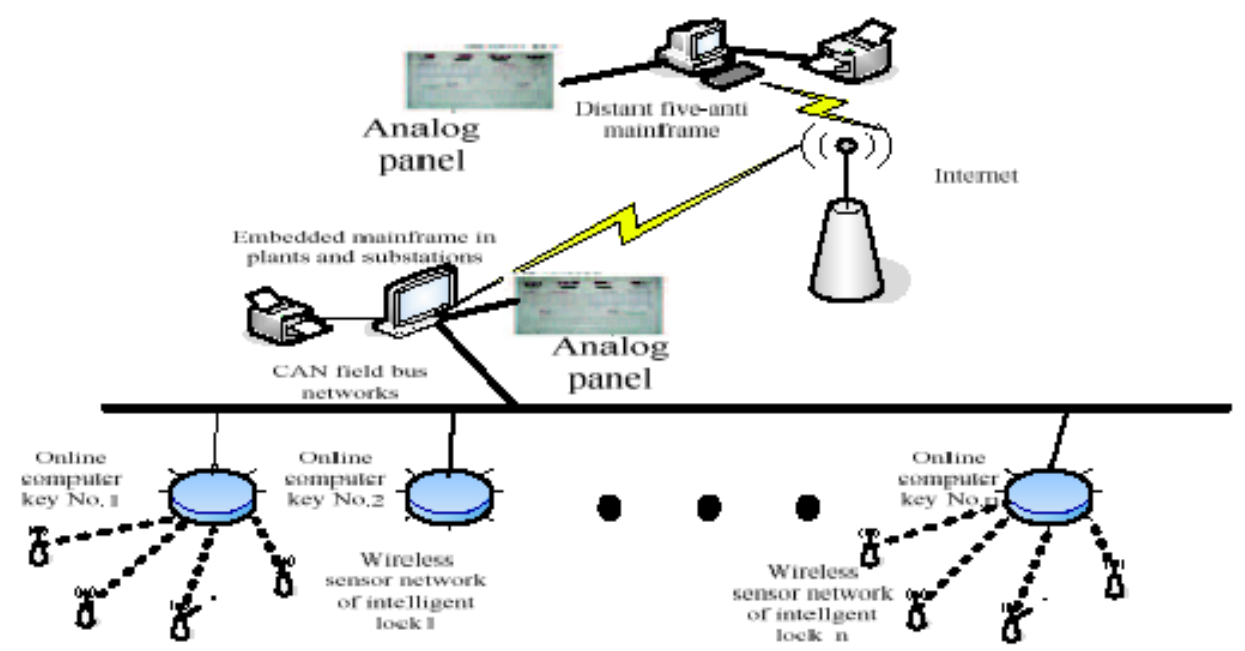

Fig.1 system framework based on multi-layer network architecture

CC2430 has small number of external components. It employs an unbalanced antenna to connect the unbalanced transformer so as to make the antenna have better function.

The parts of hardware in detail are designed as Fig.2.

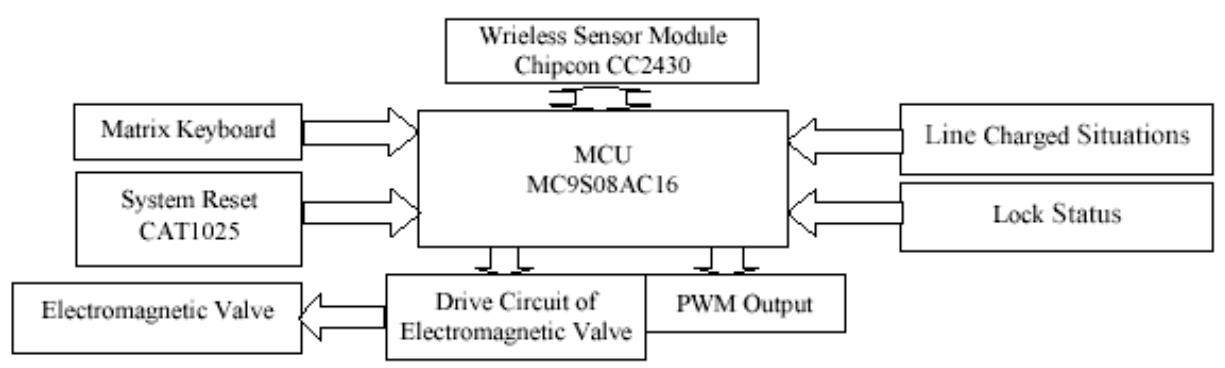

Fig.2 hardware system framework

\section{Introduction to Main VCR Technology}

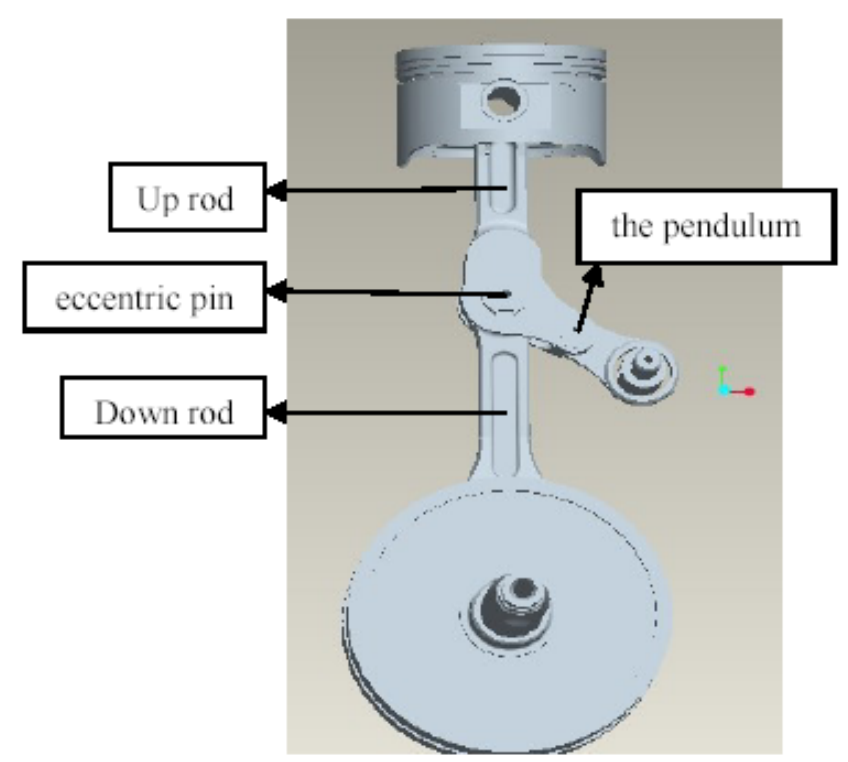

Fig. 3 the architecture of WOBAN network

The assembly drawing of this new structure is shown in Fig. 3. As shown in Fig. 3, we fix a gear on the eccentric pin; this gear meshes with the gear unit which is connected with the servomotor. 
When we need change the CR, we drive this gear unit by this servomotor, and the eccentric pin will rotate one angle, and the up rod will move up or down, finally the position of BDC and TDC will change. In our design we have chosen a servomotor with a high static torque in order to lock the eccentric pin when we didn't need change the CR. So we can ensure the reliability of the whole structure and the security of the future bench test.

\section{Comparison of New Prototype and Original Dynamic Performance}

Firstly we use the virtual prototypes that we have build to simulate the performance of these two machines, including piston displacement, piston acceleration, piston speed, piston lateral force. And then we compare these curves with the post-processing module of MSC-Adams. The analyses and comparison result is shown in Fig.4. In Fig.4 the solid line represents the new machine data; dotted line represents the original machine data. The zero point is the beginning of expansion stroke.

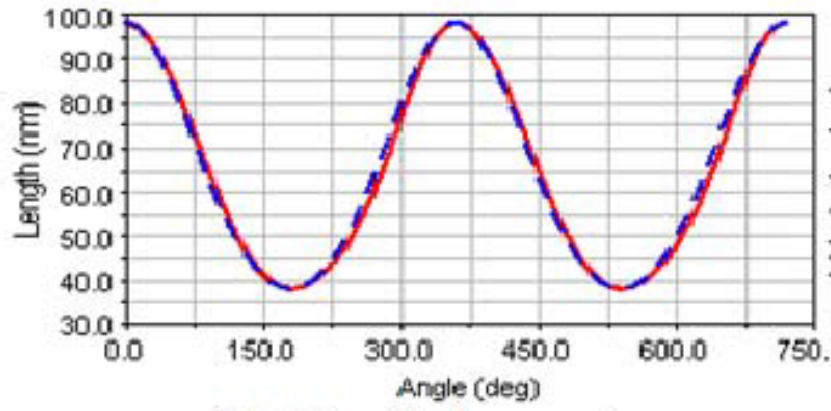

(a) piston displacement curve

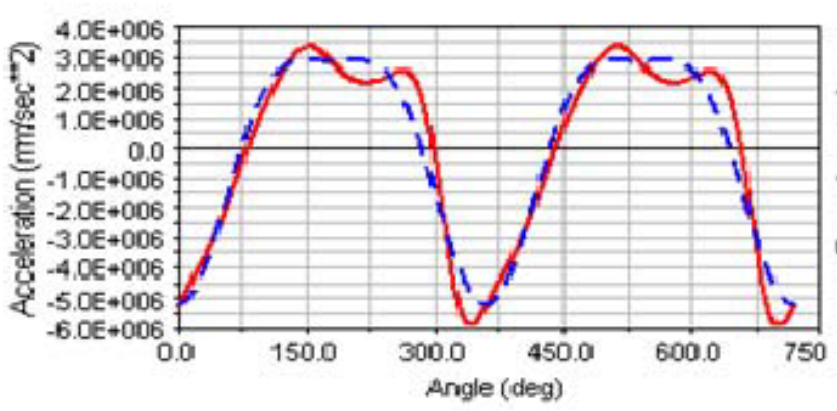

(c) piston acceleration curve

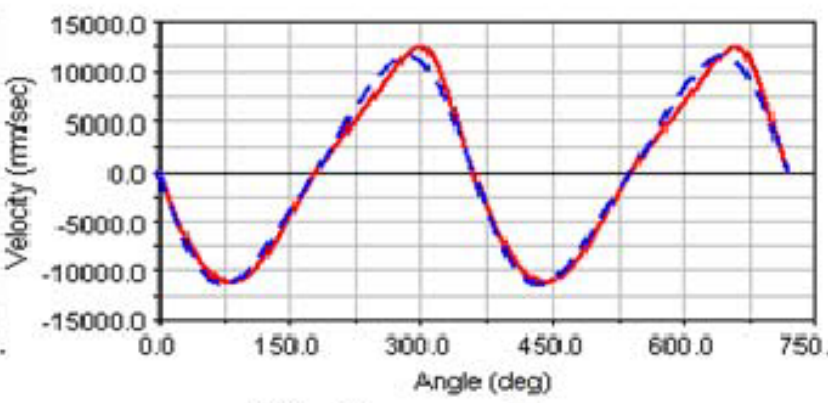

(b) piston speed curve

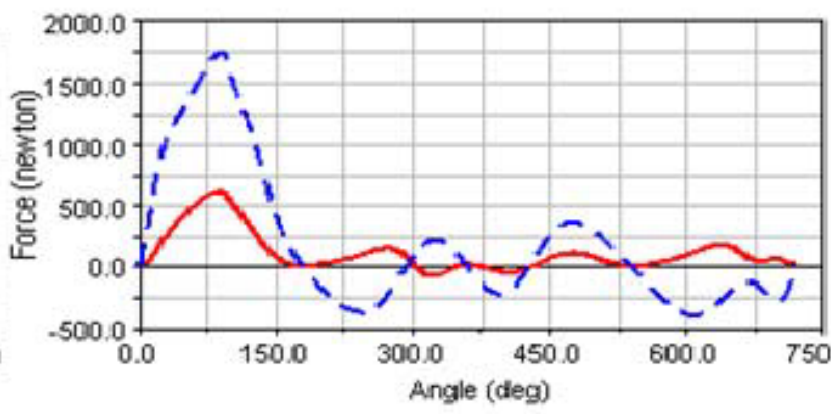

(d) pist on lateral force curve

Fig.4 the analyses and comparison results

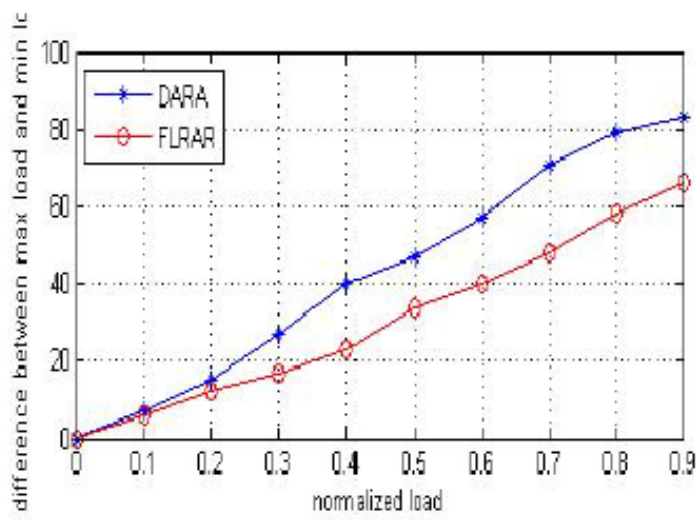

Fig.5 comparison for load balancing

If we only consider one aspect of the network constraints, other aspects of the network performance pay affect the whole performance. Take delay for example. If delay is the only factor for the algorithm, congestion will occur if most packets select one single link whose link weight is minimum for transfer. We call this load congestion. In our algorithm, we set the link capacity and LSR for each link, which ensures that the load on one link cannot cause the load congestion and 
also ensures that the network can detect the link failure on time. So FLRAR helps to achieve the load balancing in the network. We show this in Fig.5.

\section{Motion Control Section}

Fig.6 shows the spectra of Zadoff-Chu sequence respectively interpolated by FDIA and commonly used interpolation algorithm with $\mathrm{L}=1024$ and $\mathrm{k}=1$. From the figure we can see that although the spectrum of the sequence interpolated by the commonly used interpolation algorithm is compressed, it has many big side-lobes and rolls off slow. And that the spectrum of the sequence interpolated by FDIA is almost no big side-lobes and rolls off fast. So the sequence energy is centralized, which make it satisfy the bandwidth requirement of HF channel sounding transmitter-receiver.

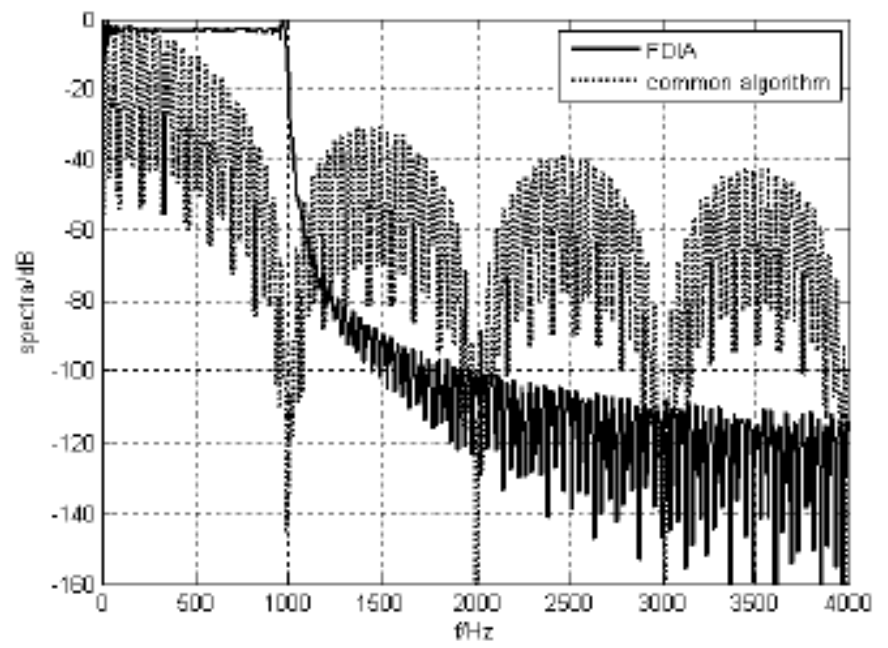

Fig.6 spectra of sequences respectively inserted by FDIA and commonly used interpolation algorithm

\section{Summary}

Compared to the original machine, the kinematic performance of the new prototype has greatly improved. Through the analysis, we can know that at every stage of operation, the new prototype perform better than the original machine. Although it has problems on vibration, we can design a uniaxial balanced device to get a better stability for the new engine.At the same time, an effective method of achieving optimal decimation of the Zadoff-Chu sequence interpolated by FDIA is provided. In the control process, it is convenient to process all necessary parameter setting and adjustment, and in the control process of the whole system shows superior performance and achieves the expected effect.

\section{References}

[1] ZHANG Jian-hua, FENG Chong, LIU Yi and ZHANG Yan-yan. "Time Synchronization Algorithm for MIMO OFDM Systems," Journal of Beijing University of Posts and Telecommunications, vol. 32, no. 1, pp. 118-121, Feb.2012.

[2] R.L. Frank and S.A. Zadoff. "Phase shift pulse codes with good correlation properties," IRE Transactions on Information Theory, vol. IT-8, pp. 381-382, Oct. 1962.

[3] D. C. Chu. "Polyphase codes with good periodic correlation properties," IEEE Transactions on Information Theory (Corresp.), vol. 18, no. 4, pp. 531-532, July 2002.

[4] K. Andrew, K. Ioannis, J. B. John, M. R. Dellomo and J. M. Sieracki. "Ambiguity and sidelobe behavior of CAZAC coded waveforms,” IEEE Radar Conference, pp. 99-103, April 2007.

[5] Zhou Longbao. ICE Principle [M]. Beijing: China Machine Press, 2007:36-37. 